Canadian Art Review

\title{
Le paysage serait-il une forme surannée ? Mise en représentation de la ville, art urbain et pratiques transitoires
}

\section{Suzanne Paquet}

Volume 35, numéro 1, 2010

Landscape, Cultural Spaces, Ecology

Paysages, espaces culturels, écologie

URI : https://id.erudit.org/iderudit/1066800ar

DOI : https://doi.org/10.7202/1066800ar

\section{Aller au sommaire du numéro}

\section{Éditeur(s)}

UAAC-AAUC (University Art Association of Canada | Association d'art des universités du Canada)

\section{ISSN}

0315-9906 (imprimé)

1918-4778 (numérique)

\section{Découvrir la revue}

Citer cet article

Paquet, S. (2010). Le paysage serait-il une forme surannée ? Mise en représentation de la ville, art urbain et pratiques transitoires. RACAR: Revue d'art canadienne / Canadian Art Review, 35(1), 32-41.

https://doi.org/10.7202/1066800ar

\section{Résumé de l'article}

This essay raises the question of the role remaining to art in today's vision and practice of landscape. Between the social demands attached to the concept of landscape and the ethical concerns linked to sustainable development, it seems that landscape has become a foreign body within artistic practice. Landscape is now often considered as an object, a piece of land shaped by human use that can even be completely reshaped by landscape architects and other professionals. Paradoxically, this point of view has been, at least in part, inspired by the work of the land artists who worked in the 1960s and 1970s: they conceived a model so strong and operative that it became a formula, and this formula has been appropriated by extra-artistic practitioners. Perhaps landscape must now be evacuated from the field of art. Certain specific instances from the Montréal context support this argument, and certain contemporary artistic interventions suggest a means of resistance to the results of the "landscaping" of cities.
Tous droits réservés @ UAAC-AAUC (University Art Association of Canada | Association d'art des universités du Canada), 2010
Ce document est protégé par la loi sur le droit d'auteur. L'utilisation des services d'Érudit (y compris la reproduction) est assujettie à sa politique d'utilisation que vous pouvez consulter en ligne.

https://apropos.erudit.org/fr/usagers/politique-dutilisation/ 


\title{
Le paysage serait-il une forme surannée? Mise en représentation de la ville, art urbain et pratiques transitoires
}

\author{
Suzanne Paquet, Université de Montréal
}

\begin{abstract}
This essay raises the question of the role remaining to art in today's vision and practice of landscape. Between the social demands attached to the concept of landscape and the ethical concerns linked to sustainable development, it seems that landscape has become a foreign body within artistic practice. Landscape is now often considered as an object, a piece of land shaped by human use that can even be completely reshaped by landscape architects and other professionals. Paradoxically, this point of view has been, at least in part, inspired by the work of the land artists who worked in the 1960s and 1970s: they conceived a model so strong and operative that it became a formula, and this formula has been appropriated by extra-artistic practitioners. Perhaps landscape must now be evacuated from the field of art. Certain specific instances from the Montréal context support this argument, and certain contemporary artistic interventions suggest a means of resistance to the results of the "landscaping" of cities.
\end{abstract}

$\mathrm{L}$ dont la définition, les attributs et les usages fluctuent à travers les siècles. De genre pictural il est devenu donné(e) sensible ou portion de territoire, puis littéralement matière à façonner. Sans vouloir refaire l'histoire du paysage en Occident, je souhaite insister ici sur sa multiplicité, sur la pluralité de ses définitions, sur toutes les possibilités d'interprétation qu'il emporte avec lui. D'abord «moyen par lequel les peintres font voir le spectacle de la nature en leur ouvre de telle manière qu'elle semble se présenter d'elle-même " 1 , le paysage s'est transformé jusqu'à devenir un produit de consommation-en quoi il s'inscrit doublement comme spectacle-, une « ressource exploitable et utilisable ${ }^{2}$ et éventuellement un objet approprié par un champ professionnel en quête de légitimité disciplinaire. Considéré à l'origine comme un tableau perspectiviste construit selon les règles du point de vue, du cadre et des relations hauteur, largeur et profondeur par lesquelles les motifs-les choses du monde perceptibles dans la portion d'espace dépeinte-sont liés entre eux, le paysage s'est muté en un artefact, soit une partie d'espace modelée par les usages, bientôt envisagée comme pouvant être re-façonnée. C'est à cette définition plus récente que je m'attacherai ici, même si, en matière de paysage, l'image et sa chose étant fréquemment confondues, la représentation n'est jamais bien loin.

En dépit de toutes ces modulations, l'idée de nature reste fortement collée au paysage. Pourtant, si c'est par la peinture qu'est né le paysage, il n'aurait rien de naturel. Particulièrement si son " invention " advient par celle de la perspective légitime, pur artifice s'il en fut, construction culturelle qui commandera éventuellement, nous dit Anne Cauquelin, une « forme de perception particulière issue d'une histoire, d'une culture, et qui fait implicitement référence à des strates de savoir que nous ne savons pas savoir ». Et, affirme encore Cauquelin, la première chose à remarquer lorsqu'il s'agit de paysage, " c'est la confusion, continûment maintenue, entre nature et paysage $»^{3}$. Pour ajouter à cette confusion, et c'est ce qui m'autorisera à associer paysage et espace public urbain en délaissant tous les autres lieux susceptibles d'être vus comme paysages ou tous les sites pour lesquels l'on pourrait revendiquer ce statut, le paysage serait, semble-t-il, un phénomène citadin. Deux raisons à cela : le paysage est né d'un besoin de nature des habitants des villes et, de nos jours surtout, c'est généralement la ville que l'on veut paysager pour répondre à diverses demandes que l'on dit sociales, dont celle de nature justement. Kenneth Clark voit dans le récit que fait Pétrarque de son ascension du Mont Ventoux en 1335 la toute première manifestation de la notion de paysage en Occident, le poète y exprimant l'émotion d'échapper aux tourments de la ville et d'accéder au repos de la campagne ${ }^{4}$. Selon toute apparence, il faut un endroit offrant un point de vue sur la nature et ce point de vue, c'est la ville 5 . Pour André Corboz, le paysage est carrément une création urbaine : "Malgré leur diversité, la poussée impressionniste, l'organisation des sports de plein air et le paysage comme spectacle ou comme expérience spirituelle, sont, encore une fois, des produits citadins, qui répondent à l'industrialisation des villes éclatées " ${ }^{6}$. De même, paysager la ville correspondrait jusqu'à un certain point à y ramener la nature, à introduire la campagne dans la ville.

Deux événements singuliers marquent, à partir des années 1960, les débuts d'un vaste phénomène de mise en paysage des villes, que j'étudierai ici : d'une part, la professionnalisation de la discipline de l'architecture de paysage et, d'autre part, l'émergence sur la scène artistique d'une nouvelle forme $\mathrm{d}$ '" art paysage ", le land art états-unien par lequel les artistes façonnent des œuvres monumentales à même des sites "naturels ", qui sont souvent des lieux désertiques et isolés. Une forme d'art dont on a d'ailleurs dit qu'elle serait apte à supplanter la peinture de paysage ${ }^{7}$. Augustin Berque signale la conjoncture : "l'œuvre d'art devenant environnement, l'art engendre l'outrepays, ce que traduisent aussi bien l'apparition de l'art-paysage aux États-Unis dans les années soixante que la diffusion, contemporaine, du terme paysagement dans les travaux publics en France " 8 . Le concept d'outre-pays peut être décrit comme une relation étroite-et assurément postmoderne-qui devrait s'établir entre l'esthétique et l'environnement, l'humain n'étant 

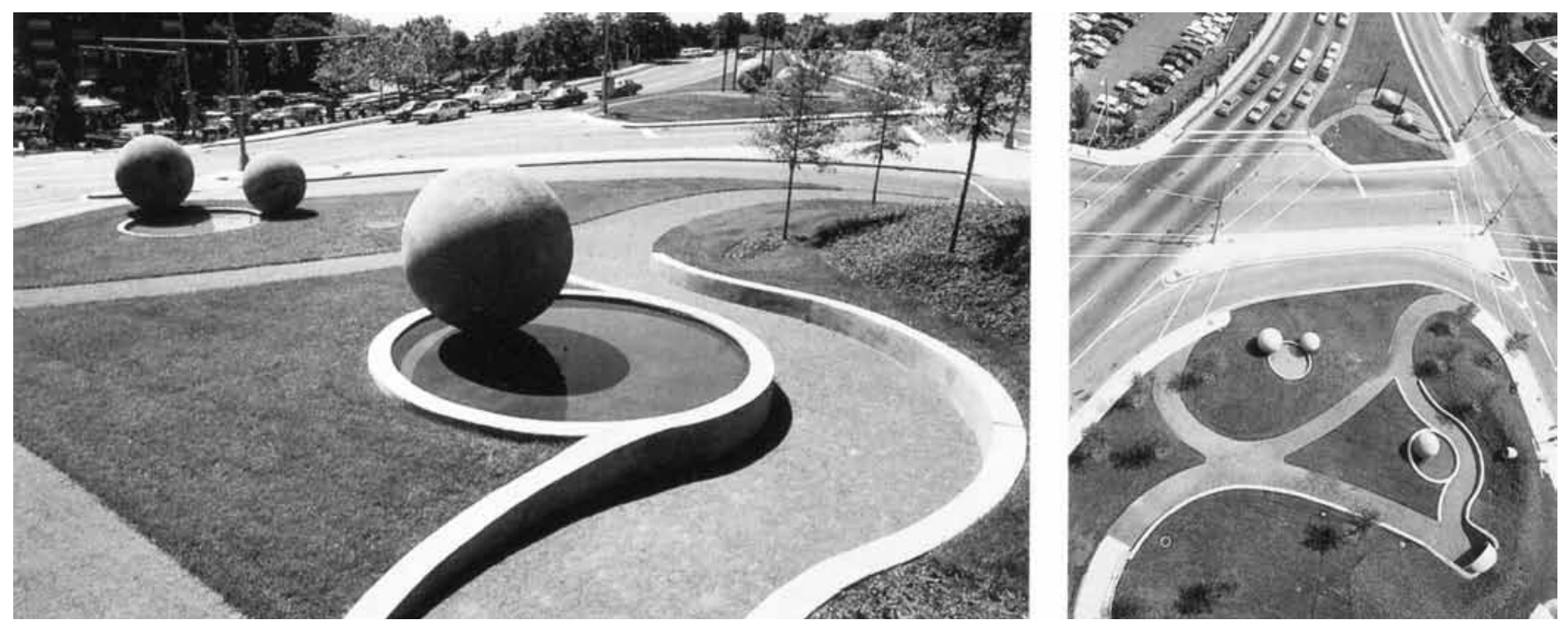

Figure I. Nancy Holt, Dark Star Park, Rosslyn, Virginie, 1979-84 (crédit photographique : Nancy Holt, reproduite ave l'aimable autorisation de l'artiste).

plus détaché de son milieu comme l'était le sujet moderne9. Fait à signaler, concurremment à la double émergence du land art et du " paysagement » se produit ce qu'Harriet Senie a qualifié de "public art revival in the late sixties ${ }^{10}$.

Au détour des années 1970, les land artists quittent les grandes étendues désolées du désert, laissant derrière eux quelques ouvres qui deviendront canoniques ${ }^{11}$, pour revenir à la ville et y prolonger leur pratique in situ en une certaine forme d'art public qui s'apparente au " paysagement " dont parle A. Berque. Puisque les habitants de la ville ont un désir de nature et que les land artists y ont pratiqué leur art, ces derniers paraissent capables de créer des œuvres qui, selon Harriet Senie, remplissent une fonction fondamentale qui consiste à procurer quelques-uns des effets réparateurs de la nature dont tous les citadins ont besoin; ces œuvres s'adressent par conséquent à une audience plus large que celle des seuls amateurs d'art ${ }^{12}$. Pour ces raisons, ces travaux d'artistes inspireront bientôt certaines formules d'aménagement des places publiques urbaines un peu partout en Occident.

Je propose donc de faire retour sur ces procédés de " paysagement " dérivés du land art, pour vérifier jusqu’à quel point ils ont influé sur la vision contemporaine de l'espace public urbain et sur la forme même du paysage. Il faudra ainsi considérer ce que devient l'art, au regard du paysage, lorsque ce dernier est approprié ou envahi par une discipline autre. Entre les implications ou les demandes sociales liées au paysage et l'éthique de développement durable qui lui est désormais attachée, quel serait le rôle de l'art? Après sa transformation artistique en un modèle si impeccable qu'il en devient une recette d'aménagement partout reprise, le paysage échapperait-il aux artistes? Ou alors, peut-être conviendrait-il d'évacuer la notion même de paysage du champ de l'art puisqu' elle y semble devenue un corps étranger. Mon propos se veut à tendance générale; quelques cas spécifiques viendront toutefois l'illustrer, dont un certain nombre d'interventions récentes dans l'espace public montréalais. Ces projets paysagers ou artistiques m'ont semblé significatifs dans la mesure où l'on pourrait en trouver de très semblables dans bien des villes occidentales.

\section{Entre nature et société}

Lorsque les land artists retournent à la ville dans les années 1970, l'art public subventionné s'oriente précisément vers des pratiques in situ que Miwon Kwon décrit comme art-as-public-spaces:

The aim was not only to accommodate the changing artistic trends of the period but to align public art more with the production of public amenities and site-oriented projects. What this amounted to in essence was a mandate for public art to be more like architecture and environmental design. ${ }^{13}$

Marquées par une forte désindustrialisation, les villes occidentales ont grandement besoin de rénovation urbaine en ces années 1970 et l'art voit son rôle social prendre de l'importance, les artistes étant appelés à participer au mouvement en faveur d'une meilleure qualité de vie citadine. Bien des artistes et des critiques veulent opposer à la seule valeur esthétique ordinairement attachée à l'art une valeur d'usage des œuvres qui témoignerait de l'engagement social de leurs créateurs. Par ailleurs, le monde de l'art ne peut faire abstraction de la question environnementale et de la montée des mouvements écologistes. Lucy 


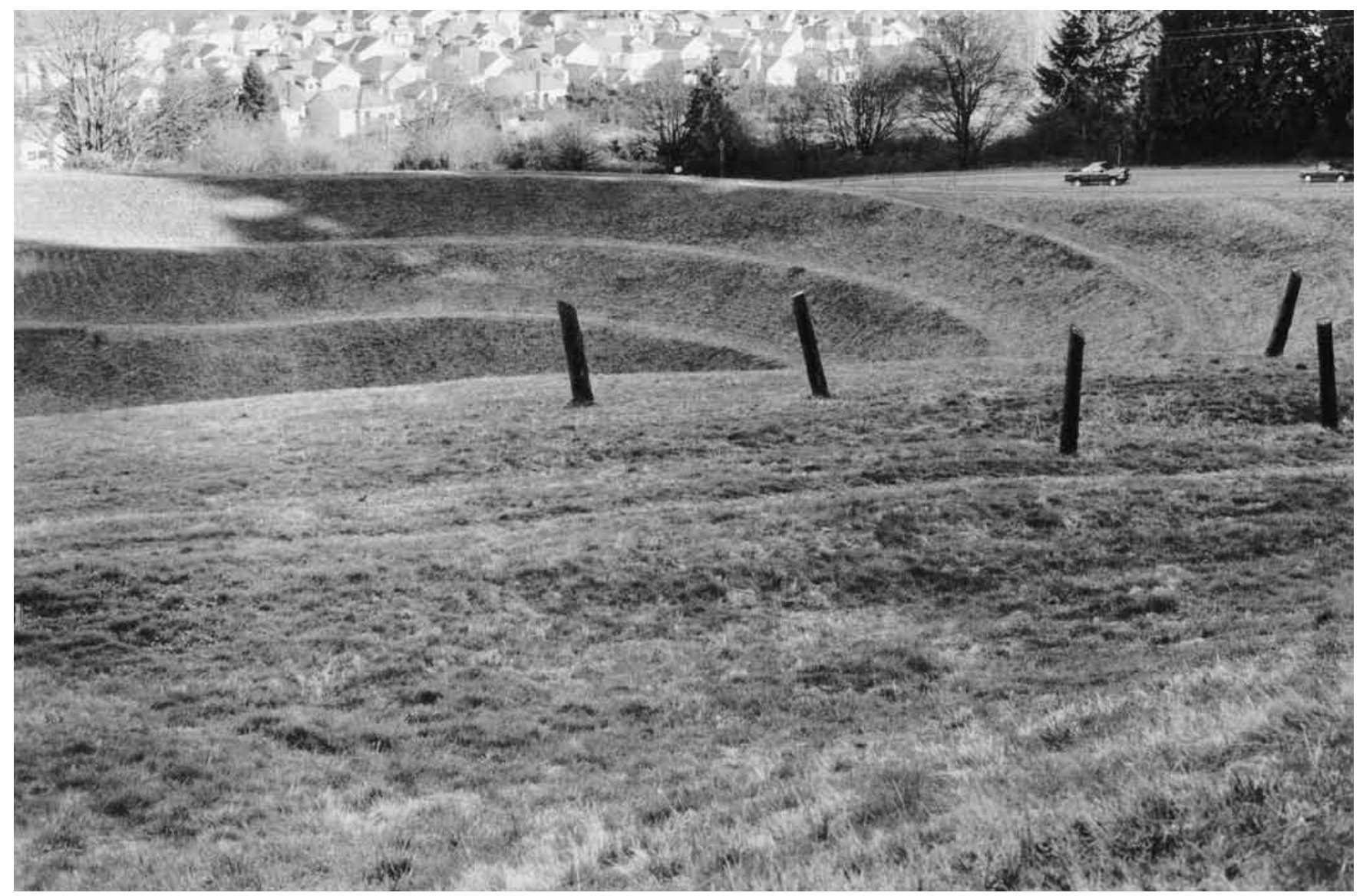

Figure 2. Robert Morris, Earthwork, Johnson Pit \#30, King County, Washington, 1979 (crédit photographique : Suzanne Paquet).

R. Lippard soutient dès 1975, dans une série de conférences et d'articles, puis dans Overlay, l'importance de la continuité entre nature, site et communauté, qui serait une nécessité pour un art vivant ${ }^{14}$. Elle défend aussi une fonction communicationnelle de l'art qui ferait qu'un art vraiment public serait celui qui ne sépare plus l'art et la vie : "In fact, public art's best chance, in this age of the corporate and bureaucratic hold on public experience, may lie in intimacy, in providing an oasis, a garden, a home in the vastness and impersonality of public contexts $» 15$. Et, suivant cette logique, Lippard affirme que le parc est vraisemblablement la forme d'art public la plus agissante qui soit, formant interface entre la nature et la société16. Le parc répond à deux besoins fondamentaux d'un art " anti-formaliste " selon Lippard : la nécessité d'une conscience écologique de la nature et celle d'une audience plus large pour l'art.

Dans certaines villes, des places publiques seront entièrement conçues par des artistes, secondés par des équipes pluridisciplinaires. L'idée est de remodeler entièrement un site dans lequel tous les éléments présents, qu'ils soient naturels ou non- y compris les éléments de mobilier urbain-, formeront une composition unique, une sorte de sculpture monumentale dans laquelle il est possible de circuler ou de s'attarder. Nancy Holt, passée du désert de l'Utah à une pratique de l'art public urbain, désigne ces œuvres comme des "parcs/œuvres d'art " (park/ artworks ${ }^{17}$. John Beardsley, dont le livre Earthworks and Beyond mêle volontiers land art, œuvres urbaines in situ et projets d'architecture de paysage, est un ardent défenseur de l'importance "sociale » d'un art public qui devrait combiner l'esthétique, le récréatif et les bienfaits de la nature en ville. Il va jusqu’à poser Dark Star Park, une œuvre de Nancy Holt construite à Rosslyn en Virginie entre 1979 et 1984 (fig. 1), comme paradigmatique d'une forme d'art public émergente, à la fois contemporaine et utile ${ }^{18}$. Cette ouvre réhabilite quelques parcelles de terrain auparavant laissées à l'abandon tout en offrant à ses usagers un espace de détente.

Autre œuvre emblématique, le Johnson Pit \#30 de Robert Morris, réalisé en 1979 à l'occasion du symposium Earthworks : Land Reclamation as Sculpture organisé par le Seattle Art Mu- 
seum, se présente aussi comme un parc public, remodelé par Morris au départ d'une carrière laissée à l'abandon depuis plusieurs années (fig. 2). Ce symposium a été l'occasion d'inviter plusieurs artistes à imaginer des projets de réhabilitation de sites désaffectés ou détériorés dans la région de Seattle. La majorité des projets proposés seront de type parc/œuvre d'art, en particulier ceux de Iain Baxter, Mary Miss, Richard Fleischner et Herbert Bayer. Un cas de ce genre peut aussi être observé à Montréal. Au début des années 1980, les artistes Charles Daudelin, Claude Théberge et Peter Gnass conçoivent l'aménagement des trois parcelles du Square Viger au centre-ville, répondant à une commande du ministère des Transports en vue de restaurer un ancien parc public mis à mal par les travaux de construction de l'autoroute Ville-Marie. Les trois artistes font des espaces qui leur sont alloués des parcs et des places, un jardin avec fontaine pour Théberge, un parc avec jeux d'enfants pour Gnass tandis que Daudelin réalise Agora, une oeuvre sculpturale qui, recouvrant entièrement son site, forme place publique (fig. 3).

Très vite, en Amérique du Nord, les architectes paysagistes vont s'inspirer des formes du land art, ainsi que de ces réalisations urbaines que sont les parcs/œuvres d'art qui sont elles mêmes, du moins dans leurs premières manifestations, le fait de land artists. En référant aux jardins et aux parcs, Lippard, Holt et leurs collègues balisent certes la voie à une appropriation de cette forme d'art public par les architectes de paysage, à un moment où la profession cherche encore une légitimité. Car, selon Françoise Dubost, c'est sa dimension artistique qui, prenant le pas sur la technique, permettra d'assurer l'« indivisible unité " du métier ${ }^{19}$. Ainsi, une certaine avant-garde chez les paysagistes revendiquera, dès le début des années 1980, le caractère artistique de leur profession, l'un d'entre eux allant jusqu'à affirmer que l'architecture de paysage est « l'art ultime parce que le paysage, social aussi bien que physique, est la source d'inspiration pour tous les autres arts ${ }^{20}$. La célèbre Martha Schwartz soutient même que les architectes paysagistes « sont allés plus loin " que les artistes du land art ${ }^{21}$. Cette supériorité déclarée ou cette indispensable emprise sur le "paysagement " des villes passe néanmoins par l'emprunt d'un modèle imaginé par les artistes, qui, entre un art pleinement intégré à son site et la réhabilitation de zones urbaines dégradées, est parfaitement adéquat, comme l'affirme Kate Linker :

Artists who in the late ' 60 s and ' 70 s had been working in remote, often desolate environments - either on private land with limited access (as with site specific sculpture) or unpeopled land (Earthworks) — brought back to the public sphere a notion of site not only easily transmissible but also highly appropriate. 22

Selon Rosalyn Deutsche, il n'est pas étonnant que ce type d'art, parce qu'il conjugue beauté, utilité et responsabilité sociale—en

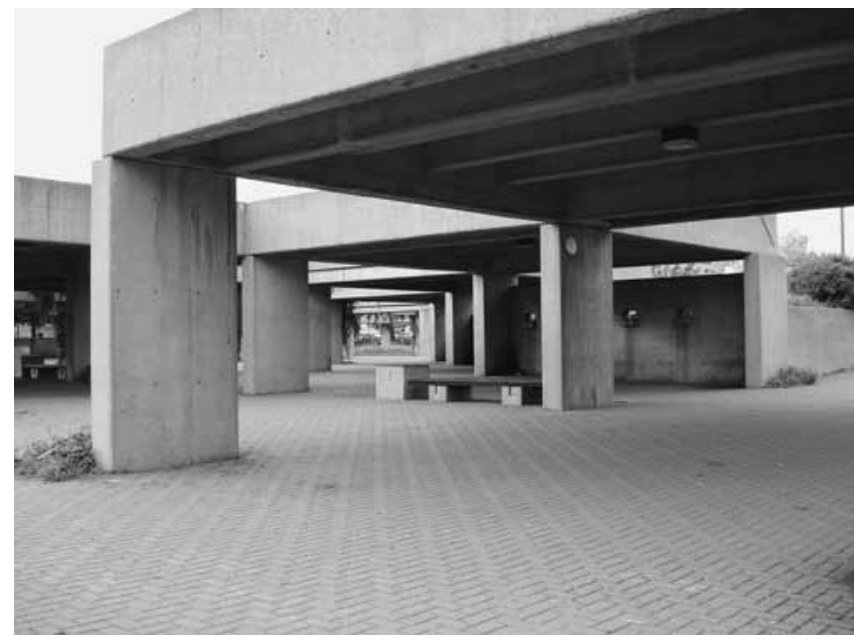

Figure 3. Charles Daudelin, Agora, Montréal, Québec, 1983 (crédit photographique : Suzanne Paquet).

donnant l'impression de rapprocher l'art et la vie quotidiennesoit étroitement lié au re-développement urbain et immédiatement repris par les praticiens professionnels du design et de l'aménagement ${ }^{23}$. En effet, le parc/oeuvre d'art véhicule un souci de l'environnement et présente une valeur d'usage, tout en étant un mode exemplaire d'esthétisation de l'espace public urbain, ce qui s'avère être une parfaite combinaison.

\section{Valeurs ajoutées}

Bien qu'ils fassent usage de la nature ou à tout le moins de sites naturels grandioses (quoique souvent hostiles), les land artists de la première génération n'ont pas vraiment de visées environnementalistes ni de propos sur la nature elle-même lorsqu'ils conçoivent les œuvres de la fin des années 1960 et du début des années 1970. Esthétiques, leurs ambitions s'articulent autour de préoccupations pour la matière, l'échelle, l'intégration de l'œuvre à son site, les relations entre l'œuvre, l'espace investi et le corps s'y mouvant. Robert Smithson, auteur de la Spiral Jetty, peut dire en 1970 :

I think we all see the desert as coextensive with the gallery. I don't think we're dealing with matter in terms of a back to nature movement. For me the world is a museum. Photography makes nature obsolete. My thinking in terms of the site and the non-site makes me feel there's no need to refer to nature any more. ${ }^{24}$

Quoiqu'on ait affirmé par la suite, le land art de la fin des années 1960 est véritablement demeuré une forme d'art pour l'art, ses tenants s'étant avant tout préoccupés de valeur esthétique. Ce sont donc d'abord des manières de faire, des visées formelles 


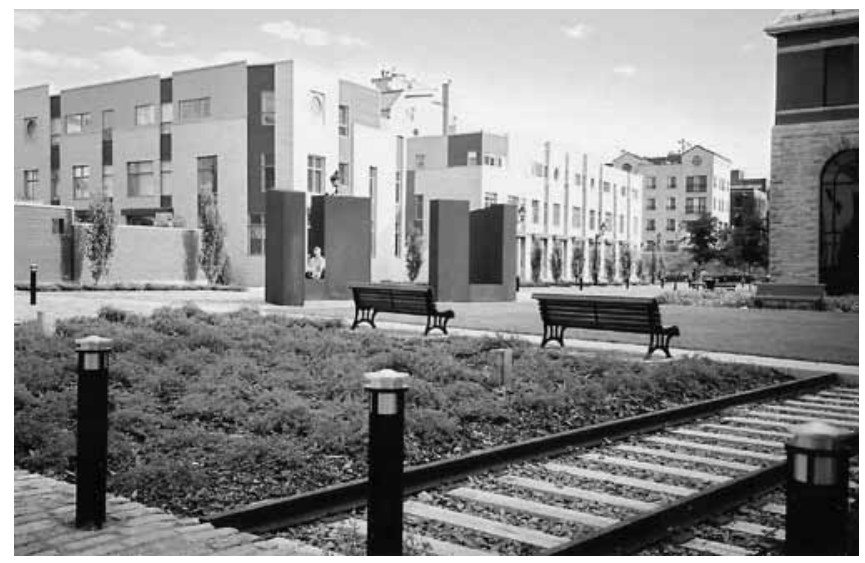

Figure 4. Jocelyne Alloucherie, Porte de jour et le Square Dalhousie, Montréal, Québec, 2004 (crédit photographique : Suzanne Paquet).

particulières, que les artistes puisent dans le land art pour les transposer à la ville. Les conditions aidant, à ces préoccupations plastiques se greffe bientôt un intérêt pour la valeur d'usage des œuvres. Le contexte social et idéologique, l'engagement et la volonté d'expérimentation artistique propres à l'époque, le rôle de la critique et aussi celui des organismes subventionnairesparticulièrement les programmes d'art public qui privilégient un art site specific proche du design-entrent en interaction pour provoquer ce glissement vers l'exigence d'une double valeur, esthétique et d'usage, des œuvres. Que l'art puisse avoir une valeur d'usage n'est rien de nouveau. Mais, après des décennies d'indépendance artistique, un retournement se produit qui se pose en rupture avec les préceptes autonomistes du modernisme: ce sont les acteurs du champ de l'art contemporain qui en appellent à une fonctionnalité des oeuvres apte à répondre aux préoccupations sociales et environnementales du lieu et de l'époque dans lesquels elles s'inscrivent. À l'inauguration de son Dark Star Park, Nancy Holt réaffirme sa conviction de l'importance (la "valeur ", dit-elle) de créer des œuvres qui sont "fonctionnelles et nécessaires à la société »25.

\section{Figures urbaines}

Simultanément aux grands mouvements de rénovation urbaine qui s'intensifient dans les années 1990 advient un fait nouveau, celui de l'interconnexion globale qui (re)mettra le paysage comme représentation au premier plan. Avec les moyens de communication électroniques, dont l'accessibilité et l'utilisation sont en forte progression, les lieux physiques, particulièrement les villes, génèrent ou deviennent des images aptes à circuler dans les réseaux transnationaux. Ainsi, dit W.J.T. Mitchell, la plupart des endroits du monde ont désormais des « jumeaux en ligne ${ }^{26}$. Tous les lieux se muent en destinations dont on reproduit les vues et perspectives pour attirer une humanité de plus en plus mobile. L'espace public urbain paysagé devient alors partie d'une valeur d'échange, un produit de consommation dont la mise en marché passe par l'omniprésence de son image, qu'il faut donc soigner : "il ne faut jamais perdre de vue que l'espace public constitue aujourd'hui un élément essentiel de la politique d'image nécessaire à la concurrence entre les villes »27, soutiennent Vlès, Berdoulay et Clarimont. La ville doit être apte à attirer visiteurs et capitaux et, à cet effet, se dessine pour nombre d'entre elles la tendance à la mise en scène ou la mise en thèmes de l'espace public. On réaménage parcs, places, rives et tout le reste de manière à illustrer le passé et l'histoire de la ville, sa haute tenue culturelle, sa qualité de vie ou n'importe quel autre aspect qui la distinguerait des autres, «la morphologie de l'espace devant faire écho aux grands thèmes structurants du récit de la ville et inversement ${ }^{28}$. Ce sont des interventions concertées dans lesquelles l'on use de façons de faire empruntées aux artistes, sans nécessairement que ceux-ci contribuent directement. C'est que la " recette " du parc/œuvre d'art reprise par les planificateurs peut aisément faire image : le land art et ses dérivés, il faut le dire, sont étonnamment photogéniques, leurs photographies se présentent généralement à l'observateur comme des tableaux de paysages majestueux.

C'est ainsi que, entre autres cas du même genre et ils sont légion, le centre-ville de Montréal devient peu à peu un assortiment de districts thématiques dont les places publiques, plusieurs incluant des œuvres d'art, projettent l'image. Quelques exemples montréalais peuvent montrer les divers rôles impartis à l'art et aux artistes dans ce récent mouvement de « thématisation » de l'espace public urbain.

La Porte de jour de l'artiste Jocelyne Alloucherie, inaugurée en 2004, est installée au Square Dalhousie du Faubourg Québec, une place publique dont l'aménagement vient rappeler les fortifications qui entouraient le secteur au dix-huitième siècle, ainsi que les voies de chemin de fer qui l'ont occupé ensuite jusqu'au moment où les opérations ferroviaires cessent en 1952 et le site est laissé à l'abandon. L'œuvre d'art est un rappel de la Porte de Québec qui perçait les fortifications du 18ième siècle (fig. 4). Selon les termes mêmes de son auteure, elle "suggère l'idée d'un mur, d'un rempart, d'une porte, évoquant par divers aspects l'histoire du lieu ». Le thème de la porte et du passage était prescrit à l'artiste par la commande, une "interprétation de la Porte Québec »étant prévue au plan d'aménagement initial ${ }^{29}$. Tous les éléments de " paysagement ", incluant l'œuvre d'art, se répondent donc de manière à produire une image du passé du site. Le Square Dalhousie dans son ensemble se présente alors comme une représentation hautement thématisée, dont la lecture est fortement orientée.

Toujours à Montréal, la Place Jean-Paul Riopelle du Quartier international intègre une œuvre du plus international des 


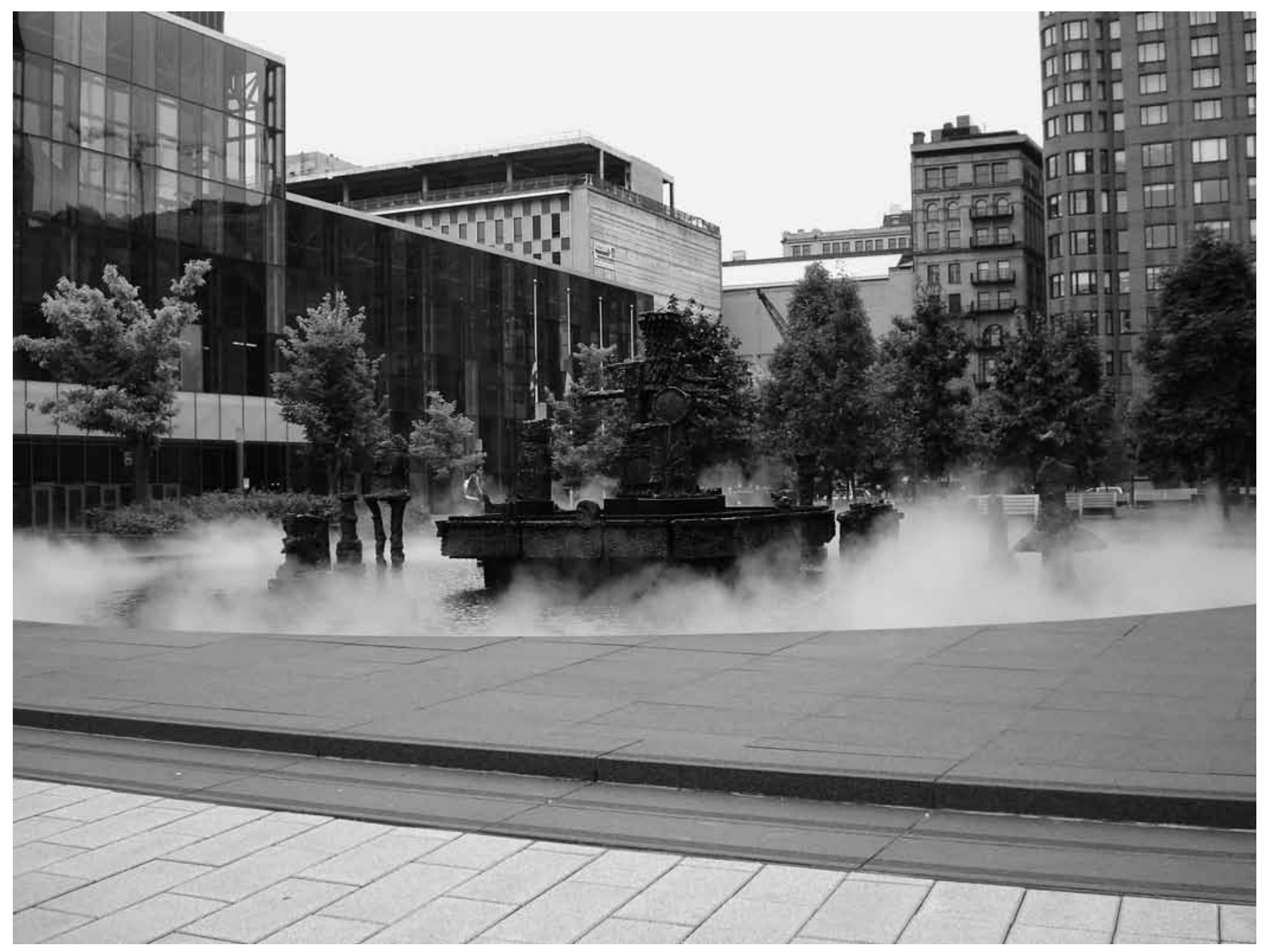

Figure 5. Place Jean-Paul Riopelle, Montréal, Québec, terminée en 2004 (crédit photographique : Suzanne Paquet).

artistes modernes du Québec. La Joute n'a pas été commandée mais plutôt déplacée du Parc olympique vers la place qui porte le nom de son créateur, elle-même spécialement configurée et équipée pour la recevoir. C'est donc l'œuvre, une fontaine qui crache des flammes et de la fumée, mais de l'eau aussi, qui détermine le thème ('internationalité) tout aussi bien que l'ensemble $\mathrm{du}$ design du lieu, selon une mise en scène saisissante : toute la place, aux moments où la fontaine en produit, est envahie par la fumée (fig. 5).

Pour le réaménagement du Square des Frères Charon, situé au croisement du Quartier international, du Vieux Montréal et de la Cité Multimédia (tous districts "à thèmes »), le Bureau d'art public de la Ville de Montréal et la Direction des parcs ont conçu un projet de collaboration réunissant un architecte, Gavin Affleck, un architecte paysagiste, Robert Desjardins, et une artiste, Raphaëlle de Groot. Dans ce cas, il n'y a pas de commande d'œuvre, ni même d'œuvre en tant que telle. On demande simplement à l'artiste de participer à la conception du projet d'aménagement. Raphaëlle de Groot n'est d'ailleurs pas connue comme une sculpteure, ni une conceptrice d'œuvres permanentes. L'interaction, les relations concrètes avec ce qui l'entoure sont au centre de sa production artistique et, selon Louise Déry :

C'est en fonction de cet agir, ou mieux de cet interagir, que Raphaëlle de Groot réalise de vastes chantiers de recherche qui l'entraînent au cœur de réalités propres à des communautés véritables $[\ldots]$. Elle s'y investit longuement, invente toutes sortes de stratégies pour en sonder les caractéristiques, multiplie les interventions auprès des individus concernés par ces milieux, collecte quantité d'éléments et d'indices révélateurs qu'elle classe patiemment et qu'elle archive de manière à constituer des corpus témoins ${ }^{30}$. 


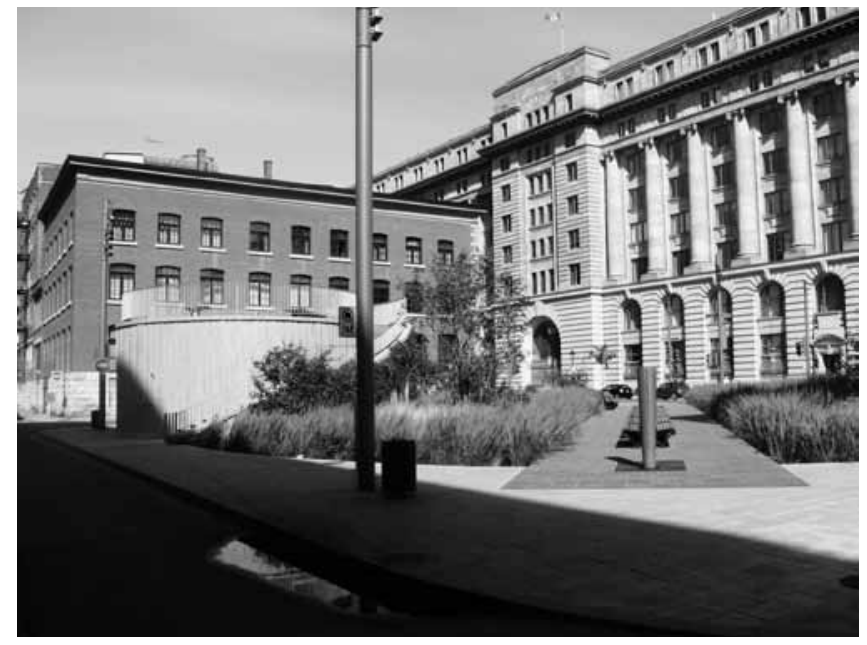

Figure 6. Square des Frères Charon, Montréal, Québec, 2007 (crédit photographique : Michelle Bélanger).

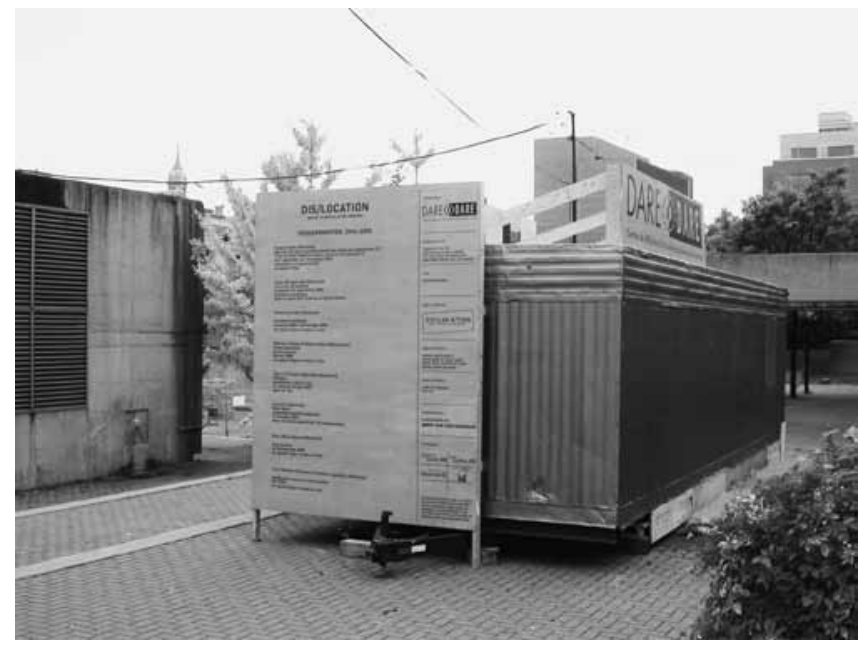

Figure 7. Abri mobile de Dare-dare à l'Agora, Montréal, Québec, 2004 (crédit photographique : Dare-dare, reproduite avec l'autorisation du centre).
Ce sont ces tactiques qui permettent à l'artiste de concevoir, sans préjuger à l'avance du support ou des matériaux, une œuvre satisfaisante et pertinente en phase avec les conditions et les lieux de l'intervention.

Les travaux au Square des Frères Charon sont complétés à l'automne 2007 (fig. 6). Dans le rendu final, on retrouve difficilement les desseins et les stratégies qui font l'originalité du travail de Raphaëlle de Groot. Comme si le "paysagement " avait eu raison d'une ébauche de réflexion particulièrement riche et caractéristique d'une artiste dont la démarche ne s'approche en rien de l'art public dans ses formes consensuelles. Le projet achevé, on retrouve encore là la mise en scène du passé comme principe directeur de l'aménagement. Si l'on en croit la description qu'en fournit la Ville de Montréal, le concept « rattache le lieu à son histoire en évoquant le paysage d'origine comme rappel de l'état naturel du site ", " une découpe circulaire réalisée à même le pavé de la ville » fait apparaître une prairie reconstituée pour remémorer le paysage "premier " de l'endroit. Mise en thème du lieu par son passé restitué et convocation de sa $n a-$ ture originelle dans la ville pour faire retour, semble-t-il, sur le vrai sens du paysage : "Le rapport fond/forme rend tangible le temps et amène à envisager la ville à partir d'un commencement qui pose le paysage et ses caractéristiques naturelles comme facteurs déterminants dans l'appropriation et l'enracinement de l'homme $» 31$. On ne saurait proposer meilleure formulation pour démontrer que le paysage est véritablement une invention urbaine et que paysager la ville correspond bien à tenter d'y ramener de la nature.

\section{Nature ou culture}

Les récents schèmes de conception des places et des squares urbains sont donc orientés vers une " thématisation " de l'espace public, une façon de proposer des villes sous forme d'impeccables figures-ce que d'aucuns appellent une stratégie de "mobilisation spectaculaire "32. La propagation de leur image, surtout celle qui ferait d'elles de hauts lieux de culture, est le meilleur instrument de mise en marché des villes-et beaucoup, dont Montréal, sont maintenant présentées comme des " métropoles culturelles ». C'est pourquoi l'espace public est lui-même reconfiguré en images, mis en tableaux. À cet égard, le modèle des parcs/œuvres d'art fournit aux aménageurs une irréprochable règle d'esthétisation de l'espace.

Ici se noue un paradoxe. Bien des théoriciens et des praticiens du domaine souhaitent pour le paysage un retour à son essence (ou sa nature) poly-sensorielle que la prédominance du visuel, effet de la modernité, aurait escamoté. Mais l'aménagement en tableaux de l'espace public des villes dément ce souhait. Et pour cause : la mise en paysage par laquelle est produite l'image propre à circuler dans les réseaux ressortit nécessairement d'une saisie frontale, à point de vue unique, qui fait retour sur la vieille formule perspectiviste à l'origine même de la notion de paysage comme objet strictement visuel.

Il y a donc dans la vaste entreprise de "paysagement " que l'Occident subit depuis quelques décennies un double effet de retournement. En un premier temps, dans les années 1960, un passage du pictural au sculptural-le land art évinçant appa- 
remment la peinture comme art du paysage-transforme, selon bien des auteurs (Berque n'est pas le seul), la définition ou les propriétés du paysage, tout comme les modalités de son façonnement. Au-delà de la représentation s'ouvre une potentialité d'esthétisation de l'espace à grande échelle. Toutefois, la mise en paysage de l'espace public urbain qui en résulte conduit rapidement à une forme de production de l'espace en images toutes frontales, à la fabrication de vues dirigées qui commandent pour ainsi dire un regard unidirectionnel, un ajustement de la vision de la chose réelle à la perspective que l'on connaissait pour l'avoir déjà vue. L'exigence de rendre la ville visible dans les réseaux entraîne une organisation spatiale faisant tableau : "surenchère du vu, le « sur-vu ", l'obscène constitutif au dispositif même qu'est le web $\$ 33$. Tout doit circuler, et tout ce qui circule doit être visuel, l'image et sa chose devant coïncider exactement, d'où l'impératif de modeler la chose en son image même. Nouveau basculement, cette fois du spatial au bidimensionnel, mais qui n'a pas été concerté par les artistes. À l'inverse de ce que souhaitait Lucy Lippard au début des années 1980, ce sont maintenant les "bureaucrates ", les planificateurs urbains, qui proposent et fabriquent parcs, jardins et paysages dans le domaine public. Tout au plus les artistes sont-ils appelés à parachever les images de marque, ces nouveaux espaces configurés comme autant de tableaux.

\section{Évacuer le paysage?}

Si Henri Lefebvre disait que l'avenir de l'art ne serait pas artistique mais urbain, il a aussi affirmé que « mettre l'art au service de l'urbain, cela ne signifie pas du tout enjoliver l'espace urbain avec des objets d'art "34. Il en appelait plutôt au sens de l'ouvre qui est " appropriation de l'espace et du temps $" 35$. Nombre d'artistes investissent ces jours-ci l'espace public urbain pour le reconfigurer temporairement ou tenter de nous en offrir une saisie différente, sans que dans leurs actions il soit question de paysage.

Peut-être serait-il temps de laisser les paysages aux paysagistes - terme qui, rappelons-le, est passé de la stricte définition de " peintres de paysages » à celle de personnes qui "notamment aménagent les espaces verts dans les villes ", dixit le Robert. Dans les villes, le paysage ne serait peut-être simplement plus le lieu de l'art ou de l'intervention artistique. Afin de suggérer la possibilité d'un certain glissement en ce sens, je me limiterai ici à décrire brièvement deux expériences artistiques montréalaises.

L'Agora de Charles Daudelin, cette sculpture formant place publique dont il a été question plus haut est, depuis son inauguration, une réalisation fort critiquée. On lui reproche son aspect bétonné, son isolement par rapport à son environnement et le fait qu'elle soit devenue un lieu de prédilection pour les personnes itinérantes du centre-ville de Montréal, pendant que,

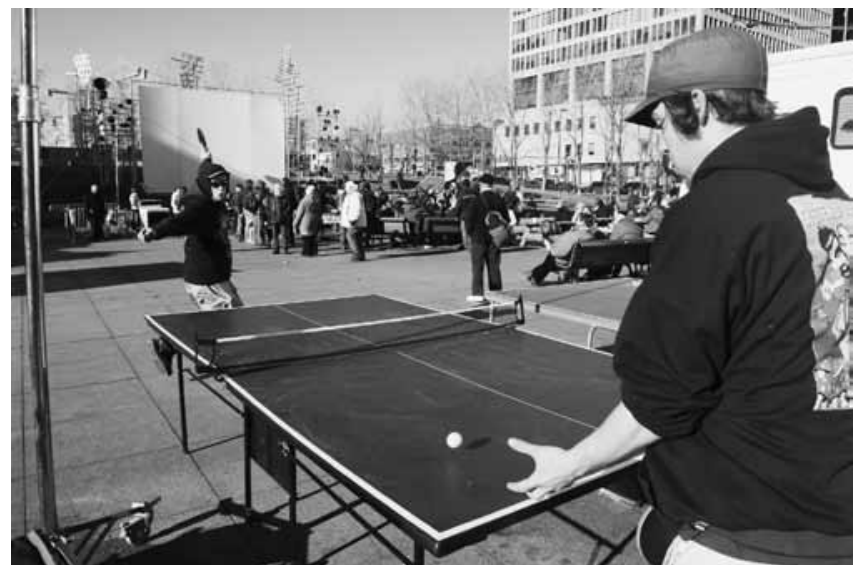

Figure 8. ATSA, État d'urgence 2007, Place Émilie-Gamelin, Montréal, Québec (crédit photographique : Martin Savoie).

pour le reste des citoyens, elle s'est transformée en une sorte de point aveugle, un endroit à éviter. En 2004, le centre de diffusion d'art multidisciplinaire Dare-dare quitte les murs de la galerie et y installe une roulotte qui demeurera sur place pendant deux ans (fig. 7). Le centre présente, à l'Agora même, une bonne partie de ses activités artistiques et, grâce à toutes ces interventions, le «public " généralement parlant ose de nouveau s'y aventurer. L'œuvre de Charles Daudelin est alors reconvertie en un véritable espace public où, par l'action de Dare-dare, la diversité des usages et des " usagers " est rétablie, le tout autorisant une certaine requalification du lieu, sans qu'il y ait la moindre velléité de « paysagement ». En 2006, Dare-dare quitte l'Agora pour s'installer dans un terrain vague du quartier MileEnd, un autre type d'expérience d'un site, l'endroit étant peu à peu converti en un parc public où les riverains circulent et s'attardent, les œuvres et les artistes y étant visibles et disponibles, tout autant que le site lui-même qui était auparavant fermé par de hautes clôtures. Dans ce lieu, encore une fois, l'action de Dare-dare modifie le site et sa perception, sans nécessiter une coûteuse opération de mise en image.

L'ATSA (Action terroriste socialement acceptable), fondée par Annie Roy et Pierre Allard, est maintenant un collectif à composition variable qui organise, entre autres événements, L'État d'urgence tenu annuellement à la Place Émilie-Gamelin. Émilie-Gamelin est un espace urbain très paysagé qui a été réalisé par une équipe de paysagistes et un artiste, Melvin Charney, dont l'œuvre se déploie dans une vaste portion de l'espace de la place. Cette oeuvre offre un bel exemple de frontalité, n'étant bien visible et clairement intelligible que d'un seul côté, d'un seul point de vue. Tout comme à l'Agora et malgré la volonté de " paysagement "—ou peut-être à cause même de la topographie 
du paysage-les jeunes sans abri du centre ville ont rapidement pris possession des lieux. Avec L'État d'urgence, à la fois camp de réfugiés urbain et "manifestival " artistique et pluridisciplinaire, ATSA occupe la place quelques jours par année, dans un esprit de cohabitation, proposant l'art comme un " moteur de la démocratie »36 (fig. 8). L'enjeu est de créer un terrain de rencontre, un lieu d'échange et de réflexion pour toutes les catégories d'" usagers ». Encore ici, c'est la notion d'espace public qui est réinstaurée, ce qu'une intervention paysagère très appuyée n'avait pas vraiment rendu possible.

\section{Écologie urbaine}

Si l'on suit encore Henri Lefebvre, on apprend que la production de l'espace peut être envisagée comme le résultat de l'interaction des pratiques spatiales, des représentations de l'espace et des espaces de représentation. Les pratiques spatiales sont le fait de l'ensemble d'une société-citoyens, gouvernants, bureaucrates, etc.-qui, agissant et s'appropriant l'espace, le modèle et lui donne forme petit à petit. Lefebvre définit les représentations de l'espace comme l'espace conçu, intellectuellement élaboré mais aussi physiquement construit par les projets, architecturaux et urbanistiques tout particulièrement. C'est l'espace « des savants, des planificateurs, des urbanistes, des technocrates 'découpeurs' et 'agenceurs', de certains artistes proches de la scientificité »37, bref de ceux qui "paysagent » les lieux. Pratiques et représentations de l'espace sont étroitement liées (l'espace contenant ses représentations et celles-ci agissant sur l'espace) et, dans le vaste mouvement de "paysagement " entrepris depuis quelques décennies, les représentations de l'espace sont conçues par les planificateurs aux fins mêmes de déterminer les pratiques spatiales et sociales dans les villes.

Au regard de ce déterminisme, un troisième terme de son schème de production de l'espace, que Henri Lefebvre nomme les espaces de représentation, attire l'attention. Ces espaces sont ceux des habitants, des " usagers " mais aussi de certains artistes : «c'est l'espace dominé, donc subi, que tente de modifier et d'approprier l'imagination »38, c'est-à-dire un espace de résistance, très clairement celui de ces artistes qui privilégient les pratiques transitoires, les interventions dans le domaine public y laissant parfois des traces mais n'ayant pas pour objectif de le modifier durablement, à tout le moins dans son aspect physique. Il y a là un souci de l'environnement qui dépasse largement l'idée de paysage et s'approche d'une écologie urbaine qui tient compte du milieu et contient du social, qui intègre les habitants des lieux 39 . On l'a souvent dit d'ailleurs, un paysage n'est pas quelque chose que l'on habite mais bien une fonction perceptive qui demande de la distance ${ }^{40}$.

" [L'espace de représentation] contient les lieux de la passion et de l'action, ceux des situations vécues, donc implique immédiatement le temps. De sorte qu'il peut recevoir diverses qualifications : le directionnel, le situationnel, le relationnel, parce qu'il est essentiellement qualitatif, fluide, dynamisé. »41 Comment mieux dire? Tous ces termes utilisés (en 1973, il faut le souligner) par Henri Lefebvre sont en prise directe avec les récentes pratiques et les actions urbaines des artistes. Envahissant l'espace des planificateurs, ces lieux recomposés en tableaux, en paysages, ils arrivent à leur donner un sens autre, que chacun peut, s'il le veut, s'approprier pour ainsi re-créer de véritables espaces publics.

Dans la mesure où le paysage comme artefact est désormais l'objet d'un champ disciplinaire qui n'a rien à voir avec l'art et que son exercice est une opération planifiée et planificatrice très souvent imposée à vrai dire, la pratique artistique peut très bien s'en détacher. Les citadins auront toujours besoin de naturecomme d'équité. Mais le paysage n'est peut-être plus la notion opportune, ni la solution toute trouvée.

Notes

1 Anne Cauquelin, "Le paysage n'est pas un lieu ", Paysages sur Commande, Rennes, Le Triangle, 1990, p. 94. Je souligne.

2 Georges Cazes, Robert Lanquar et Yves Raynouart, L'aménagement touristique, Paris, Presses universitaires de France, 1993, p. 34.

3 Anne Cauquelin, op. cit., p. 93. Voir également, de la même auteure, L'invention du paysage, Paris, Presses universitaires de France, 2002 (1989).

4 Kenneth Clark, Landscape into Art, Boston, Beacon Press, 1963 (1949), p. 1.

5 Roland Recht, La Lettre de Humboldt, Paris, Christian Bourgeois, 1989, p. 57.

6 André Corboz, Le Territoire comme palimpseste et autres essais, Paris, Éditions de l'imprimeur, 2001, p. 224.

7 Voir : Colette Garraud, L'Idée de nature dans l'art contemporain, Paris, Flammarion, 1994.

8 Augustin Berque, Médiance. De milieux en paysages, Montpellier, Reclus, 1990, p. 132.

9 Ibid., p. 128-39.

10 Harriet F. Senie, Contemporary Public Sculpture. Tradition, Transformation and Controversy, New York, Oxford University Press, 1992, p. 14.

11 Entre autres : la Spiral Jetty (1970) de Robert Smithson, le Double Negative (1969) de Michael Heizer, Las Vegas Piece (1969) de Walter De Maria et les Sun Tunnels (1973-76) de Nancy Holt.

12 Harriet Senie, op. cit., p. 170 et p. 16.

13 Miwon Kwon, One Place After Another. Site-Specific Art and Locational Identity, Cambridge, MIT Press, 2002, p. 67. Rosalyn Deutsche décrit ainsi cette forme d'art : "The new public art was defined as art that takes the form of functional objects placed in urban spaces_-plumbing, park benches, picnic tables_or as art 
that helps design urban spaces themselves ". Evictions. Art and Spatial Politics, Cambridge, MIT Press, 1996, p. 259.

14 Lucy R. Lippard, Overlay. Contemporary Art and the Art of Prehistory, New York, Pantheon Books, 1983, p. 238. Je traduis, ainsi que pour les autres citations d'écrits anglais intégrés à ce texte, afin d'alléger la lecture.

15 Lucy R. Lippard, "Gardens : Some Metaphors for a Public Art ", Art in America, novembre 1981, p. 139. Je souligne.

16 Lucy R. Lippard, Overlay, op. cit., p. 228.

17 Joan Marter, "Nature Redux: Recent Reclamation Art ", Sculpture, novembre-décembre 1994, p. 32.

18 John Beardsley, Feuillet de présentation Dark Star Park, Rosslyn, Arlington County, Virginia, 1984, n.p.

19 Françoise Dubost, "Les paysagistes et l'invention du paysage ", Sociologie du travail, no. 4-83, 1983, p. 437.

20 Garett Eckbo cité par Catherine Howett, "Landscape Architecture : Making a Place for Art ", Places, vol. 2, no. 4, 1985, p. 58.

21 Brenda Brown, "Avant-Gardism and Landscape Architecture ", Landscape Journal, vol. 1, no. 10, p. 145. Encore en 2000, John Beardsley déclare : "I wish to make an extreme statement, if only to make an empathic one: landscape architecture will prove the most consequential art of our time ". John Beardsley, "A Word for Landscape Architecture ", Harvard Design Magazine, no. 12, automne 2000, p. 5.

22 Kate Linker, "Public Sculpture : The Pursuit of Pleasurable and Profitable Paradise ", Artforum, mars 1981, p. 66-67. Je souligne.

23 Rosalyn Deutsche, op. cit., p. 66.

24 Liza Bear et Willoughby Sharp, « Dicussions with Heizer, Oppenheim, Smithson " (1970), dans Jack Flam (dir.), Robert Smithson : The Collected Writings, Berkeley, University of California Press, 1996, p. 246.

25 Texte de Nancy Holt, Feuillet de présentation Dark Star Park, Rosslyn, Arlington County, Virginia, 1984, n.p. Dans le même feuillet, cette déclaration de John Beardsley : "I suggested that some narrative or symbolic content on some purposeful—utilitarian-intent could transform contemporary art in public places, often awkward in its unfamiliar new home, into an acceptable contemporary form of public art."

26 William J.T. Mitchell, "Wunderkammer to World Wide Web : Picturing Place in the Post-Photographic Era ", dans Joan M. Schwartz et James R. Ryan (dir.), Picturing Place. Photography and the Geographical Imagination, New York, I.B. Tauris, 2003, p. 299.

27 Vincent Vlès, Vincent Berdoulay et Sylvie Clarimont (dir.), Espaces publics et mise en scène de la ville touristique, rapport final de recherche, Ministère délégué au tourisme, Direction du tourisme, Université de Pau et des Pays de l'Adour, Laboratoire SET, octobre 2005.

28 Ibid., p. 74.

29 Documents relatifs au réaménagement du Square Dalhousie, Bureau d'art public, Ville de Montréal, 2003.
30 Louise Déry, "L'exposition mise en exercice ", Raphaëlle de Groot. En exercice, Montréal, Galerie de l'UQAM, 2006, p. 27.

31 Tous les extrait cités proviennent de: http://www.vieux.montreal. qc.ca/charon/description.htm.

32 Timothy A. Gibson, « La ville et le « spectacle » : commentaires sur l'utilisation de "Spectacle " dans la sociologie urbaine contemporaine ", Sociologie et Sociétés, vol. XXXVII, no. 1, printemps 2005, p. 176.

33 Antoine Hennion "CyberMeb ", Sociologie et Sociétés, vol. XXXII, no. 2, 2000, p. 17 (version électronique : http://www.erudit.org/ revue/socsoc/2000/v32/n2).

34 Henri Lefebvre, Le Droit à la ville, suivi de Espace et politique, Paris, Anthropos, 1968, p. 140 et 139.

35 Ibid., p. 138 et 139.

36 http://www.atsa.qc.ca

37 Henri Lefebvre, La Production de l'espace, Paris, Anthropos, 2000 (1973), p. 48.

38 Ibid., p. 49.

39 «La question sociale est donc au centre des préoccupations de l'écologie urbaine. Il s'agit de fabriquer la cohésion sociale en ne séparant pas les problèmes de justice sociale et culturelle et ceux de justice environnementale. Une grande importance est accordée à la question de l'espace public dans ses dimensions tant philosophiques qu'urbanistiques, à la réappropriation de la ville par le citadin pour qu'il en devienne citoyen. "Vincent Berdoulay et Olivier Soubeyran, L'Écologie urbaine et l'urbanisme. Aux fondements des enjeux actuels, Paris, La Découverte, 2002, p. 25.

40 Voir: Denis Cosgrove, Social Formation and Symbolic Landscape, Madison, University of Wisconsin Press, 1998 (1984), p. 18-19. Également : "To what extent does a place-image created for external audiences correspond to the insiders' sense of place, if indeed there is a consensus among the latter? "Cara Aitchison, Nicola E. Macleod et Stephen J. Shaw, Leisure and Tourism Landscapes, Londres, Routledge, 2000, p. 153.

41 Henri Lefebvre, La Production de l'espace, op. cit., p. 52. 